\title{
Stable Disease by Computed Tomography
}

National Cancer Institute

\section{Source}

National Cancer Institute. Stable Disease by Computed Tomography. NCI Thesaurus.

Code C123615.

The size and extent of tissue involvement by cancer is neither decreasing nor increasing, based on CT scan and criteria-defined assessments. 